\title{
Molecular states of two vertically coupled systems of classical charged particles confined by a Coulomb potential
}

\author{
F. F. Munarin, ${ }^{1}$ W. P. Ferreira, ${ }^{1, *}$ G. A. Farias,${ }^{1}$ and F. M. Peeters ${ }^{2, \dagger}$ \\ ${ }^{1}$ Departamento de Física, Universidade Federal do Ceará, Caixa Postal 6030, Campus do Pici, 60455-760 Fortaleza, Ceará, Brazil \\ ${ }^{2}$ Department of Physics, University of Antwerp, Groenenborgerlaan 171, B-2020 Antwerpen, Belgium
}

(Received 20 February 2007; revised manuscript received 7 May 2007; published 26 July 2007)

\begin{abstract}
We numerically study the structure and the normal-mode spectrum of two vertically coupled finite-size two-dimensional systems of interacting classical charged particles that are confined by a nonuniform distribution of background charge. The structural and dynamical properties of the system are analyzed as a function of the separation between the planes of charges $(d)$, as well as a function of the strength of the confinement potential $(Z)$. We find different asymmetrical ground-state configurations, as well as structural phase transitions induced by the unbinding of particles, which were not present in previous models with a parabolic confinement potential. Depending on the order of the structural transition the normal-mode frequencies exhibit a discontinuity or a softening.
\end{abstract}

DOI: 10.1103/PhysRevB.76.035336

PACS number(s): 36.40.Ei, 64.90.+b, 83.10.Tv

\section{INTRODUCTION}

A systematic study of the structural and dynamical properties of the classical version of the two-dimensional (2D) quantum dot (also called artificial atoms) has been made in recent years. ${ }^{1-6}$ Such a classical approach of the problem serves as a good starting point for more demanding quantummechanical calculations. At low temperatures and in the presence of high magnetic fields, electrons in quantum dots become localized and can be approximated as pointlike charges. In this limit, some structural and melting properties of the original quantum system can already be obtained through a classical approach. ${ }^{7}$ The classical $2 \mathrm{D}$ system is usually modeled as $N$ pointlike charges confined by an external potential, which is most often chosen to be the parabolic one and which is equivalent to a uniform background of neutralizing charge.

For a small number of particles the symmetry of the parabolic confinement potential is imposed over the triangular structure as favored by the repulsive Coulomb interaction between the charges. As a result, a circular shell structure is observed as ground state. ${ }^{1,2,7}$ However, if a different confinement potential, e.g., resulting from a nonuniform neutralizing background, is considered, the electrostatic correlations between particles become more important, especially in the limit of a weak confinement potential. ${ }^{5,8}$ In such a situation, spontaneous symmetry breaking and very interesting asymmetrical ground-state configurations as well as a nontrivial melting behavior were predicted. ${ }^{5,8,9}$

Motivated by the increased interest in laterally coupled quantum dots ${ }^{10-15}$ and by the fact that many properties of quantum systems can be already observed in a classical approach, the behavior of the classical double-layer system of charged particles was recently studied for both vertical and horizontal types of coupling cases. ${ }^{16,17}$ Due to the twodimensional nature of the system, the choice of vertical or horizontal coupling will lead to different structural properties of the system. Once more, we stress that in the previous classical approaches only a parabolic type of confinement was considered. In a first step such a confinement is very suitable, but it is also worth commenting that some interesting physical properties appear only when corrections are added to the parabolic potential-e.g., the violation of the Kohn theorem ${ }^{19}$ and an observable anticrossing behavior in the power absorption spectrum. ${ }^{20,21}$

We extend our previous work ${ }^{5,8}$ to vertically coupled dots and concentrate on the effects due to a nonparabolic type of confinement potential on the structural and dynamical properties. The present model system is not completely academic but it can be related to the "superatom" system introduced by Watanabe and Inoshita. ${ }^{22}$ The superatom is a circular modulation-doped heterojunction. In particular, it is a quasiatomic system that consists of a circular donor-doped core and a surrounding impurity-free matrix with a larger electron affinity. The quantum-mechanical electron structure of this system was studied in Ref. 23, and it was found that due to the absence of the $1 / r$ singularity in the potential, the ordering of the energy levels is dominated by the no-radial-node states, in contrast to real atoms, where $s$ and $p$ states are dominant.

Apart from the above-mentioned analogy with quantum dots in the classical limit, there exist other experimentally realized systems that behave purely classically and for which our study is relevant. Examples are charged colloidal suspensions where it was found recently that correlation effects between the counterions can result into an overscreening and attraction between like-charged colloids. ${ }^{18,24}$ In addition, we can also cite dusty plasmas and magnetic colloids ${ }^{25-27}$ where 2D dotlike structures have been realized.

This paper is organized as follows. In Sec. II, we describe the mathematical model and our numerical approach to obtain the configurations and frequencies of the normal modes. The results and discussions of our numerical simulations are given in Sec. III. Our conclusions are presented in Sec. IV.

\section{MODEL}

Our system consists of $2 \mathrm{~N}$ negatively charged particles, which we label now as electrons, evenly distributed in two planes or layers which are separated at a distance $d$. The 


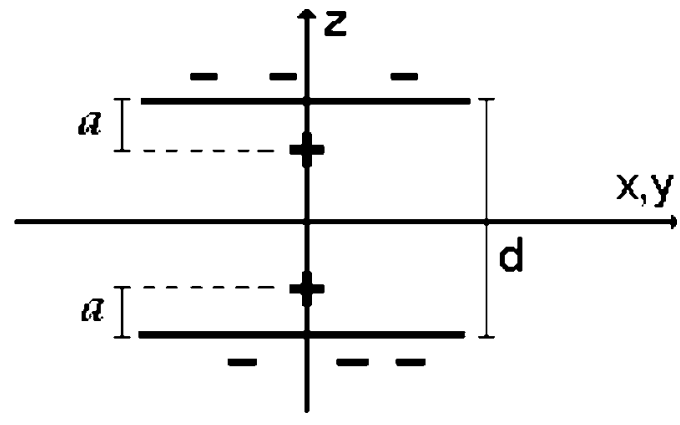

FIG. 1. Schematic view of the model system where the positive confinement charges are placed in the internal region between the planes.

electrons in each layer are kept together by two positive charges (each one equal to $Z$ ) which are located between those two planes. A schematic view of the system is shown in Fig. 1. The positive charge is displaced a distance $a$ from the electron plane and is assumed to be the same for both electron layers. From now on we label this system as the Coulomb confined molecule (CCM).

The potential energy of the CCM system is given by

$$
\begin{aligned}
H= & -\frac{Z e^{2}}{\epsilon}\left(\sum_{i=1}^{N} \frac{1}{\sqrt{r_{i}^{2}+a^{2}}}+\sum_{i=1}^{N} \frac{1}{\sqrt{r_{i}^{2}+(a-I)}}\right) \\
& +\frac{e^{2}}{\epsilon}\left(\sum_{\substack{i>j=1 \\
(I, I I)}}^{N} \frac{1}{\left|\mathbf{r}_{i}-\mathbf{r}_{j}\right|}+\sum_{i=1}^{N} \sum_{j=1}^{N} \frac{1}{\left|\mathbf{r}_{i}-\mathbf{r}_{j}+d^{2}\right|}\right),
\end{aligned}
$$

where $(I, I I)$ represent the sums over particles in the respective layers. Here the symbol $\epsilon$ stands for the dielectric constant, $\mathbf{r}=\{x, y\}$ is the two-component position vector in $2 \mathrm{D}$ space, and $\left|\mathbf{r}_{i}-\mathbf{r}_{j}+d^{2}\right|$ represents the distance between the electrons in the distinct electron planes.

We introduce units for the energy $E_{0}=e^{2} / \epsilon a$ and distances $a$. This allows us to rewrite Eq. (1) in the dimensionless form

$$
\begin{aligned}
& H=-\sum_{i=1}^{N} \frac{Z}{\sqrt{r_{i}^{2}+1}}-\sum_{i=1}^{N} \frac{Z}{\sqrt{r_{i}^{2}+(I I)}}+\sum_{\substack{(I, I I) \\
i>j=1}}^{N} \frac{1}{\left|\mathbf{r}_{i}-\mathbf{r}_{j}\right|} \\
&+\sum_{i=1}^{N} \sum_{j=1}^{N} \frac{1}{\left|\mathbf{r}_{i}-\mathbf{r}_{j}+d^{2}\right|} . \\
& \quad \text { (I) (II) }
\end{aligned}
$$

The ground-state configurations of the system were obtained through the standard Metropolis algorithm (at zero temperature) combined with the modified Newton method to increase the accuracy of the ground-state energy. ${ }^{2}$ The numerical procedure considered in this paper allowed us to obtain a different number of stable states, which depends on $N$ and $Z$. From all the obtained stable states, the one with lower energy was taken as the ground-state configuration. To check if a configuration is stable, we calculated, using the Householder diagonalization technique, the eigenvalues of the dynamical matrix,

$$
H_{\alpha \beta, i j}=\sum_{i=1}^{N} \frac{\partial^{2} H}{\partial r_{\alpha, i} \partial r_{\beta, j}},
$$

which give us the square of the frequencies of the normal modes of the system $(\alpha, \beta=x, y$ and $i, j$ indicates the particle number). The configuration was taken as final when all frequencies of the normal modes were positive. When $N \ll Z$ we showed before ${ }^{5,6}$ that the structural and dynamical properties of the single-layer Coulomb-confined system become the same as the ones observed for the classical parabolic confined dot. ${ }^{1}$ We also found that the unit of frequency is proportional to the square root of the confinement charge-i.e., $\omega_{0}=\sqrt{e^{2} / m \epsilon a^{3}} \sqrt{Z / 2}{ }^{6}$ In the present paper we use $\omega_{0}$ for the unit of frequency.

\section{RESULTS AND DISCUSSIONS}

The structure and the normal-mode spectrum of the system will be studied as a function of the distance $(d-2)$ between the confinement positive charges and the strength of the confinement potential $(Z)$. As mentioned before, in the limit $Z \gg N$ the single-layer Coulomb-confined system behaves similar to the previously studied parabolic-confined system. ${ }^{1}$ We found that this is also true when we consider the present system in the same limit and with the confinement charges in the region outside the planes of electrons. The vertically coupled system confined by a parabolic potential was studied previously by Partoens et al. ${ }^{16}$

For the configuration shown in Fig. 1, we will focus on the neutral $(Z=N)$ and the underconfined $(Z<N)$ cases. In these limits the electronic correlation effects are the most pronounced and dominate the physical properties of the system. We find that the main features of the present model can already be observed if we consider the small, but nontrivial cluster with $2 N=6$ particles. However, results for clusters with different numbers of particles will also be presented for completeness.

In order to better observe how the ground-state configuration is affected by the distance between layers or, more precisely, between the two Coulomb-confined atoms $(d-2)$, we investigate the distance of each particle from the $z$ axis $\left(r_{i}\right)$ as a function of $(d-2)$. The dependence of the structural properties on the intensity of the confinement potential will also be considered.

We start showing $r_{i}$ as a function $(d-2)$ for the cluster with $2 N=6$ particles in the neutral case $2 Z=6$. Notice that all particles in both layers have the same value of $r_{i}$, which depends on $(d-2)$. The corresponding ground-state configuration is an equilateral triangle in each layer, and the two triangles are rotated with respect to each other in order to maximize the interlayer particle distances. Note that the size of the triangles increases with increasing value of $(d-2)$, which is a consequence of the weaker interaction between the confinement charge $(\mathrm{Ze})$ of one layer and the particles in the other plane. The behavior of $r_{i}$ shown in Fig. 2(a) indicates that for a sufficiently large value of $(d-2)$ the distances of all particles to the $z$ axis approaches a constant value. The long-range nature of the Coulomb interaction implies that the 


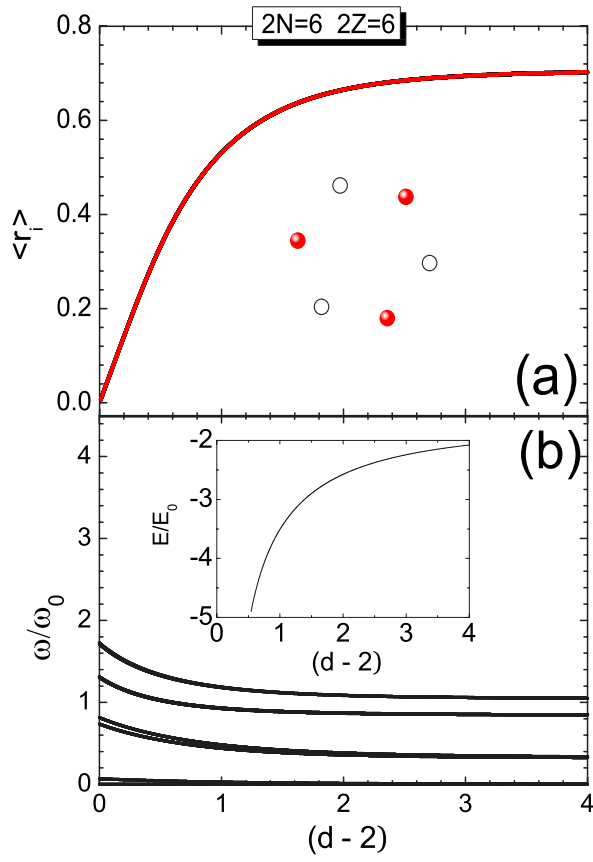

FIG. 2. (Color online) (a) Distance of each particle from the $z$ axis as a function of the separation between the positive charges $(d-2)$, for a system with $2 N=6$ and $2 Z=6$. (b) Normal-mode frequencies as a function of $(d-2)$ for the same system. The energy of the minimum-energy configuration as a function of $(d-2)$ is shown in the inset of (b). An example of the ground-state configuration is presented in the inset of (a). Open and solid circles represent particles in different layers.

system will never be independent, but for a sufficiently large value of $d$ the contribution of the interaction between particles in distinct planes is small enough not to influence the configuration (and the energy) of the separate layers. For example, for the case shown in Fig. 2(a), we found that for $(d-2) \geq 3.5$ the distance of each particle from the $z$ axis is already $r_{i} \approx 0.707$, which is the same distance observed in the single-layer system with $N=3$ and $Z=3.5,6,8$

The energy as a function of $(d-2)$ is shown in the inset of Fig. 2(b). No structural phase transition is observed when $d$ is changed, implying that the first and second derivatives of the energy with respect to $(d-2)$ are continuous. Thus the system continuously transits from a moleculelike behavior, where there is a strong coupling between the particles on the distinct layers, to an atomlike behavior, where the contribution of the Coulomb interaction between particles in distinct layers is very small.

The normal-mode spectrum, as a function of $(d-2)$, for the same cluster is shown in Fig. 2(b). For large values of $(d-2)$ the normal-mode frequencies become the same as the ones of the single system with $N=3$ and $Z=3$. In the opposite case-i.e., for small separation between layer-some of the degeneracies are lifted. The behavior of the normal-mode spectrum corroborates the idea that the planes of particles behave like independent systems for large values of $(d-2)$. The particular arrangement of the positive charges shown in Fig. 1 screens the interaction between particles in distinct layers. Only for small values of $(d-2)$ does such an interac-

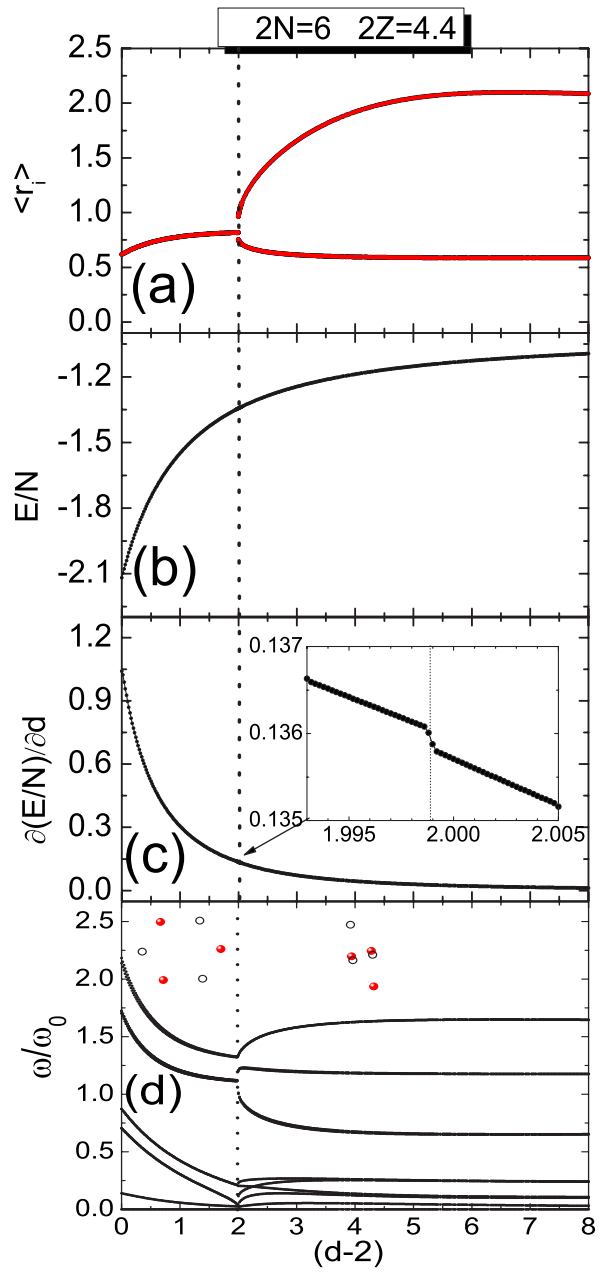

FIG. 3. (Color online) (a) The distance of each particle from the $z$ axis, (b) the energy, (c) the first derivative of the energy with respect to $(d-2)$, and (d) normal-mode frequencies as a function of $(d-2)$ for a cluster with $2 N=6,2 Z=4.4$. Examples of the groundstate configuration of the system are presented as inset in (d). Open and solid symbols represent particles in distinct layers.

tion become strong enough to modify the system.

According to Ref. 8, a stable single-layer Coulombconfined system with $N$ electrons is only possible if the condition $Z>N-1$ is met. If this requirement is not fulfilled, one or more electrons become unbound; i.e., they move to infinity. The situation $N>Z>N-1$ is referred to as the overscreened case.

The sketch presented in Fig. 1 suggests that the electrons in each plane are confined by both positive charges for any finite distance $d$. However, the effective interaction between the electrons and the positive confinement charges depends on $d$. We can argue that the electrons in each layer are confined by the charge $2 Z$ only when $(d-2)=0$. If $d>2$, the Coulomb interaction between electrons in one layer and the positive charge of the other layer is smaller than the interaction between these electrons and their own confinement charge, in order that the effective confinement charge of the electrons in each layer is smaller than $2 Z$, but still larger than $Z$. Note that when $d \rightarrow \infty$ the effective confinement charge for the electrons in each layer becomes $Z$, since the Coulomb 
interaction between electrons and the second confinement charge becomes negligible. Thus, for finite values of $d$, such a confinement charge is effectively larger than $Z$.

Again, we stress that the arrangement presented in Fig. 1 suggests that it is possible to obtain stable ground-state configurations by considering, for instance, a system with $2 N$ $=6$ electrons and $2 Z=4.4$. In this case, the effective confinement charge for the electrons in each layer is $Z_{\text {eff }}>2.2$, which is, in principle, in agreement with the condition given in Ref. 8. However, if we consider that such a criterion should take into account all charges in the system, as done in Ref. 8, then we find that the condition $2 Z>2 N-1$ is no longer obeyed.

The results for the distance of each electron $\left(r_{i}\right)$ from the $z$ axis as a function of the separation between the confinement charges $(d-2)$ are presented in Fig. 3(a). As can be observed, for $(d-2) \lessgtr 1.999$, the negative particles of both planes stay at the same distance from the $z$ axis, forming an equilateral triangle in each plane [see the inset in Fig. 3(c)]. For $(d-2) \approx 1.999$, a sudden structural change takes place in the system [see the inset in Fig. 3(c)]. Such a change is associated with a first-order structural phase transition, which is confirmed by Fig. 3(c) where a discontinuity of the first derivative of the energy with respect to $(d-2)$ is observed at $(d-2) \approx 1.999$. The energy is continuous for all values of $(d-2)$. Note that all particles are bounded (i.e., confined) for all values of $(d-2)$. The excess of negative charge (overcharge) in the system is $\Delta q=1.6$, which is larger than the allowed overcharge in the one-layer system. ${ }^{8}$ The overcharge is due to the screening (by the positive charges) of the interaction between electrons in distinct planes. We found that when the positive charges are placed at the region external to the layers, there is no screening in the interaction between electrons in distinct layers and the overcharge is always $\Delta q<1$, as in the single-layer system. Note that the electrons in distinct planes could be seen as counter-ions adsorbed on the surface of a large colloidal particle. The present model already demonstrates overcharging, ${ }^{18}$ which here is a consequence of the screening of the interaction between the counter-ions.

Now we present some other interesting and nontrivial features of the present model. To do so we choose some particular distances $d$ between layers and we study the system as a function of the confinement charge $Z$. In general, we found that asymmetric ground-state configurations and spontaneous symmetry breaking can appear as function of $(d, Z)$. When $Z$ is sufficiently decreased to confine a certain number of particles, one electron may suddenly leave the system and go to infinity. Such an unbinding process or "evaporation" of particle is characterized by a first-order structural phase transition.

As an example, the average distance of each particle from the $z$ axis $\left(r_{i}\right)$ and the frequencies of the normal modes as a function of $Z$ are presented in Fig. 4 for the cluster with $2 N \leqslant 6$ particles. The distance between the positive charges is chosen to be $(d-2)=2$. We define in Fig. 4 nine different regions which are associated with distinct ground-state configurations, separated from each other by first- or secondorder structural transitions.

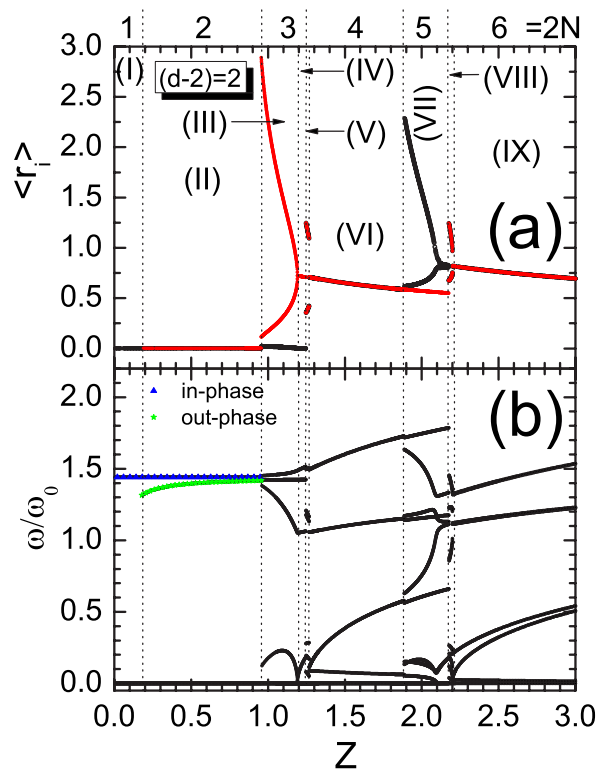

FIG. 4. (Color online) (a) The distance of each particle from the $z$-axis as a function of $Z$ for a cluster with $2 N \leqslant 6$, and $(d-2)=2$. Black and red curves represent the positions of particles in distinct layers. On top of this figure the number of confined particles is given. (b) The normal mode frequencies as a function of $Z$. The frequencies of the in-phase (blue circles) and the out-of-phase (green stars) vibration of the particles around the center of the confinement charge in each plane, Eqs. (5) and (6), respectively, are also presented.

We start with the region (IX) $(Z \geqslant 2.201)$, where particles in each layer are located at the vertices of an equilateral triangle. Viewing from the top [Fig. 5(a)], the equilateral triangles are orientated with respect to each other, forming a hexagon, which indicates a correlation between particles sitting in distinct layers. When $Z=2.201$ the system suffers a first-order structural phase transition to region (VIII) (2.175 $\leq Z<2.201$ ). Such a transition is characterized by an abrupt change of the positions of the particles as a function of $Z$. The new ground state configuration is shown in Fig. 5(b). The particles in each layer are now located at the vertices of isosceles triangles, which are oriented with respect to each other. The intensity of the confinement potential is not strong enough to keep all particles at the same distance, which means that the electrostatic correlations between the electrons already start to dominate the structure of the cluster since the circular symmetry of the confinement potential is no longer reflected in the symmetry of the ground-state configuration.

For $Z=2.175$ another abrupt structural transition takes place, leading the system to region (VII) $(1.857 \leqq Z$ $<2.175$ ), where now the configurations in each layer are different [Fig. 5(c)]. The transition from region (VIII) to region (VII) is characterized by the unbinding of a single particle. Such an asymmetrical evaporation process results in a spontaneous symmetry breaking of the ground-state configuration of the entire system. The ground-state configuration observed in region (VII) is also observed in regions (IX) and (VIII), but as a metastable state. As an example, for $Z$ $=2.22[$ region (IX)] the ground-state configuration [Fig. 5(a)] 

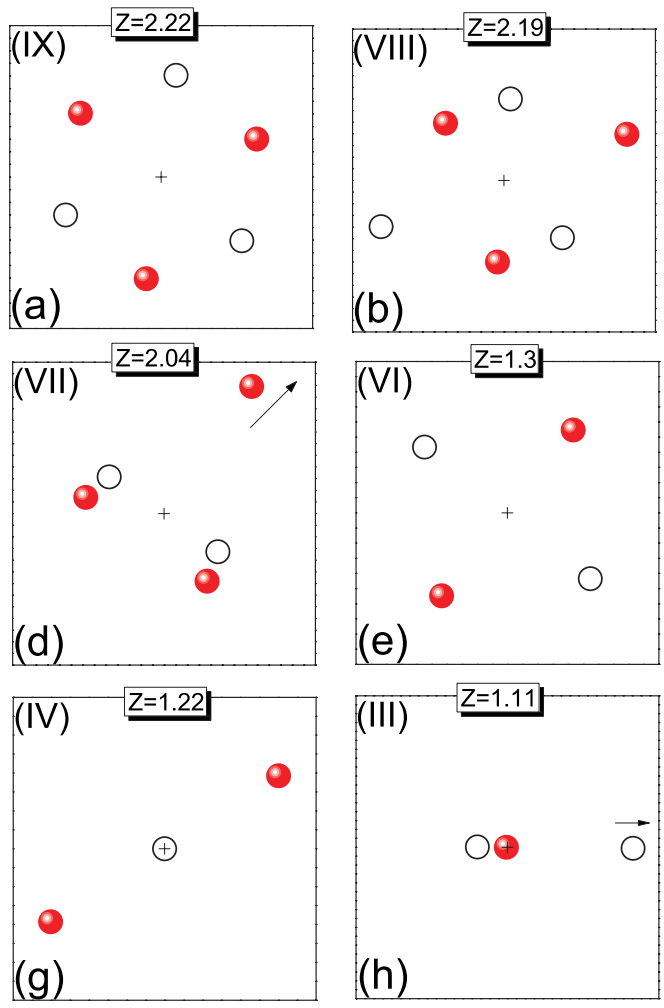

FIG. 5. (Color online) Top view of the ground-state configurations of the system with $2 N \leqslant 6$ and $(d-2)=2$ in the different regions of Fig. 4. Particles in distinct layers are represented by different symbols (open black circles and closed red spheres). The $z$ axis is located by the black cross. is observed with energy $E_{\text {ground }}=-1.365853$, while the metastable state corresponding to the configuration shown in Fig. 5 (c) has energy $E_{\text {meta }}=-1.359902$. Notice that the groundstate configuration is determined by the competition between the attractive confinement potential and the repulsive interaction potential between the negative particles. In this case, the unbinding of one particle results in a larger confinement energy for the remaining particles, resulting in a larger energy of the entire system.

When $Z$ is further decreased in region (VII) the configuration changes continuously in order to reach a more symmetrical arrangement of the particles. As a consequence, one electron of the layer with $N=3$ leaves the system in a continuous way up to $Z=1.857$ [Fig. 5(d)], where it suddenly becomes unbound. The system is now in region (VI) $(1.268 \leqq Z<1.857)$ with a very symmetrical minimumenergy configuration and with two particles in each layer [see Fig. 5(e)]. The evaporation of two particles makes again the confinement potential the dominant term imposing the symmetry of the ground state.

For $Z=1.268$ a new first-order structural phase transition takes place, leading the system to the small region (V) $(1.247 \leqq Z<1.268)$, where a very nontrivial ground-state configuration is observed [Fig. 5(f)]. It is interesting to notice that in spite of the evenly distributed number of particles in the planes, the configuration is asymmetric; i.e., particles in each layer are located at different distances from the $z$ axis. The symmetrical configuration observed in region (VI) is still obtained as a stable state in region $(\mathrm{V})$, but with higher energy. For example, for $Z=1.26$ the ground-state configuration [Fig. 5(f)] has energy $E_{\text {ground }}=-0.561026$, while the symmetrical configuration similar to the one observed in region (VI) appears as a metastable state with energy $E_{\text {meta }}=-0.560911$.
For $Z=1.247$ the system reaches region (IV) $(1.192 \leqq Z$ $<1.247)$ through a new first order structural phase transition, which is associated with the unbinding of one more particle. The system has now a different number of particles in each layer [Fig. 5(g)]. The asymmetrical number of particles in the layers observed in region (IV) occurs only over a very short interval of $Z$. For $Z=1.192$ the ground-state configuration changes rapidly and continuously, which is a secondorder structural transition. Such a transition is characterized by the softening of one of the normal-mode frequencies [see Fig. 4(b)]; i.e., one of the normal-mode frequencies becomes zero at the transition point.

The system now is in region (III) $(0.956 \leq Z<1.192)$, where another particle starts to leave the system, becoming unbound at $Z=0.956$, through a new first-order structural transition to region (II) $(0.190 \leqq Z<0.956)$, where both particles are sitting on the top of its respective positive charge. A further decrease of the confinement charge generates a new first-order structural phase transition to region (I), in which only one particle remains on top of its positive charge. This occurs for $Z \approx 0.190$. In general, we found that the value of $Z$ corresponding to such a transition is given by the expression

$$
Z=\frac{d-1}{d^{2}},
$$

which is obtained by making the energy of the system with two particles equal to the energy of the system with only one particle.

The corresponding normal-mode frequencies of the configurations are presented in Fig. 4(b). In general, the normalmode frequencies are more degenerate when the ground state 

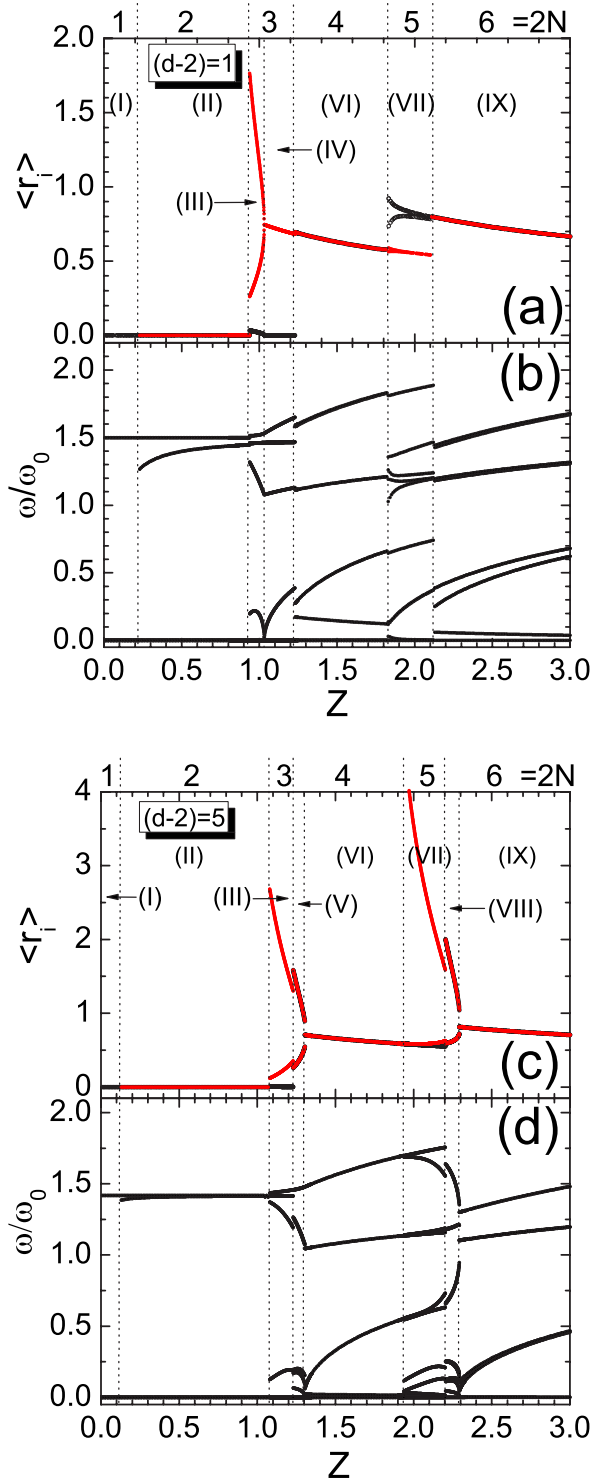

FIG. 6. (Color online) The same as Fig. 4 but now for (a), (b) $(d-2)=1$ and $(\mathrm{c}),(\mathrm{d})(d-2)=5$.

is symmetric. These modes are very sensitive to first- and second-order structural phase transitions. For first-order structural changes the frequencies exhibit abrupt jumps at the transition points, while for second-order transitions there is a softening of one of the modes. ${ }^{15}$

For all values of $Z$ there is one mode with $\omega=0$, which is associated with the rotation of the entire system around the $z$ axis. The normal-mode frequencies depend on the strength of the confinement potential. There is an exception in region (II), where a nonzero mode with constant frequency is observed. This is the in-phase vibration of the particles around the center of the confinement potential-i.e., the center of mass (c.m.) mode. Note that in region (II) there is another mode which decreases with decreasing value of $Z$, and it is the out-of-phase c.m. mode; i.e., the particles in distinct planes oscillate always in the opposite direction around the $z$ axis. A simple calculation gives us, respectively, the following expressions for the in-phase and for the out-of-phase vibration of the c.m. mode:

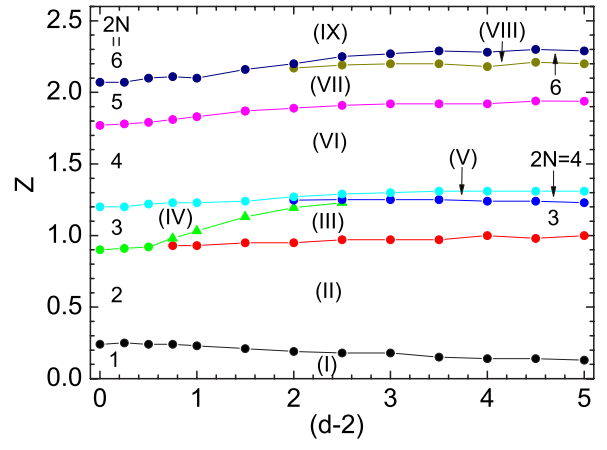

FIG. 7. (Color online) Phase diagram $Z \times(d-2)$ for the groundstate configurations of the system with $2 N \leqslant 6$. The number of particles, $2 N$, in each region is also presented. First- and second-order structural phase transitions are represented by circles and triangles, respectively.

$$
\begin{gathered}
\omega_{\text {in }} \approx \sqrt{Z+\frac{Z}{(d-1)^{3}}}, \\
\omega_{\text {out }} \approx \sqrt{Z+\frac{Z}{(d-1)^{3}}-\frac{2 N}{d^{3}}} .
\end{gathered}
$$

The frequencies obtained from Eqs. (5) and (6) are, respectively, shown in Fig. 4(b) as blue triangles (in-phase vibration) and green stars (out-of-phase vibration). Due to the nature of the confinement potential, which is associated with a nonhomogeneous charge distribution, there is not always a center-of-mass mode for any number of particles in the system, in contrast to the parabolic-confined case. In region (I), there is only one particle and its motion is the same as the one of the center of mass.

As suggested by Eq. (2) the physical properties of the system are also ruled by the distance between the layers $d$. To illustrate this we show in Fig. 6 results for different distances between the confinement charges: namely, $(d-2)=1$ and $(d-2)=5$. The regions labeled in these figures correspond to the same configurations shown before in Fig. 5. As can be seen in Figs. 6(a) and 6(c) some configurations observed for $(d-2)=2$ (Fig. 4) are now absent. For example, for $(d-2)=1$ the configurations associated with regions (VIII) and (V), in Fig. 5, are not observed. The cluster transits directly from region (IX) [Fig. 5(a)] to region (VII) [Fig. $5(\mathrm{c})]$ and from region (VI) [Fig. 5(e)] to region (IV) [Fig. $5(d)$. In both cases there is evaporation of one charge, which leads the system to an unbalanced distribution of charges over the layers. The same is observed for $(d-2)=5$, but now the unbinding of one particle leads the system directly from region (V) [Fig. 5(f)] to region (III) [Fig. 5(h)].

The distance between layers also influences the normalmode spectrum. In general, clusters with larger distance between layers present a more degenerated normal-mode spectrum due to the smaller interaction between charges of distinct layers. This fact can be seen, e.g., in Figs. 6(b) and $6(d)$. For instance, notice that in region II the out-phase vibration of the center-of-mass mode in the case $(d-2)=5$ is 

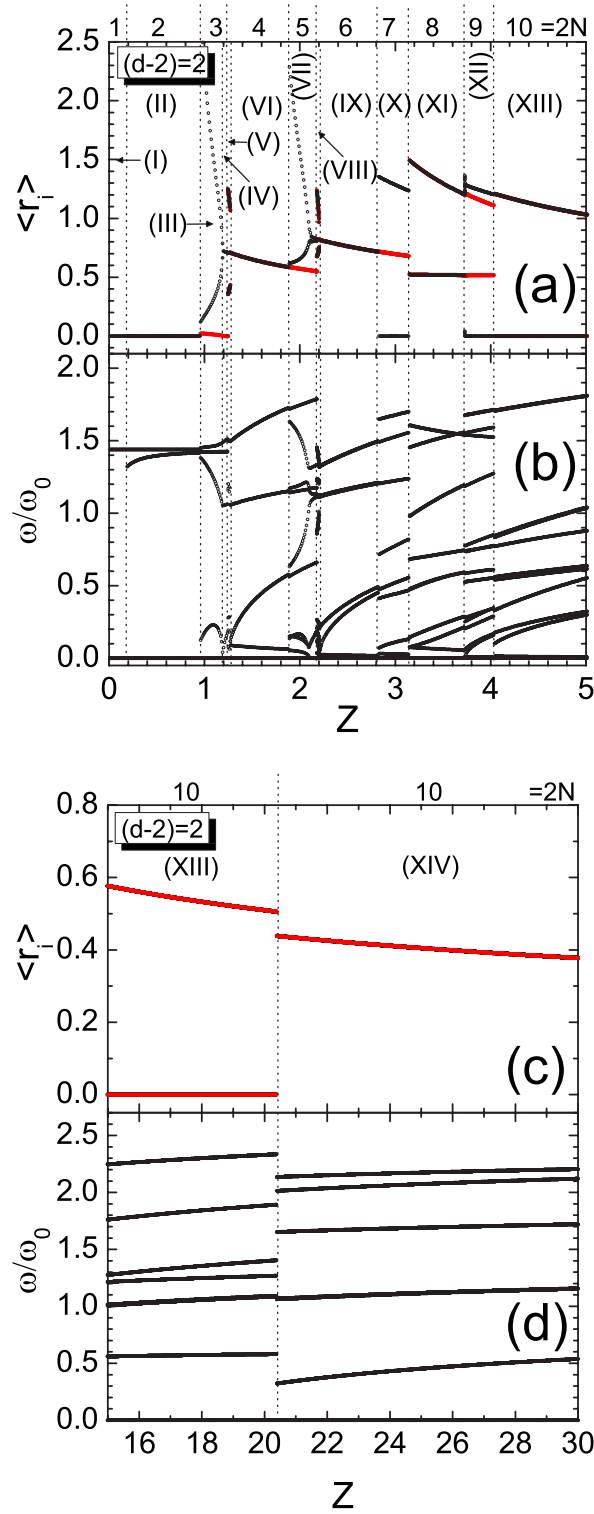

FIG. 8. (Color online) (a),(c) The distance of each particle from the $z$ axis as a function of $Z$ for the cluster with $2 N \leqslant 10$ and with distance between the confinement charges $(d-2)=2$. Black and red curves represent the positions of particles in distinct layers. On top of these figures the number of confined particles is given. (b),(d) The normal-mode frequencies as a function of $Z$ for the same cluster.

much less sensitive to $Z$ than the same mode in the case ( $d$ $-2)=1$, where the interaction between particles in distinct layers is stronger.

The general dependence of the ground-state configurations of clusters with a maximum number of confined charges $2 N \leqslant 6$ on the parameters $Z$ and $(d-2)$ is summarized in the phase diagram $Z \times(d-2)$, presented in Fig. 7 . The stability range of the different configurations depends on the parameters $(Z,(d-2))$. For example, the ground-state configuration associated with region (VIII) of Fig. 5 is observed only for $Z \gtrsim 2$. Configurations (III), (IV), and (V) are only obtained for some values of $(Z,(d-2))$. The transitions between the different regions-i.e., configurations-occur
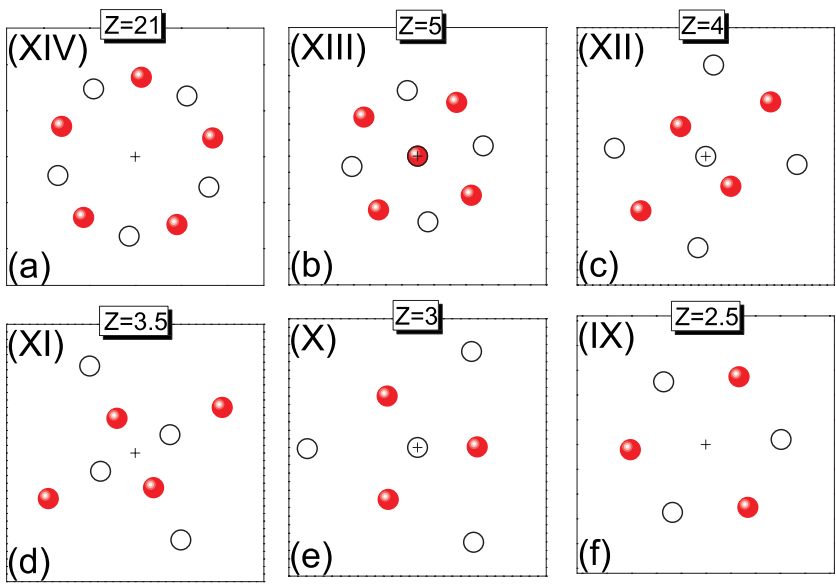

FIG. 9. (Color online) Top view of the ground-state configurations of the system with $2 N \leqslant 10$ and $(d-2)=2$ for different regions of Fig. 8. Particles in distinct layers are represented by different symbols (open black circles and solid red spheres). The center of the system is given by the black cross.

most often through first-order structural transitions. The specific case of structural changes induced by the evaporation of one or more particles is always characterized by a first-order structural transition. Second-order structural changes were also obtained, but only between configurations (III) and (IV) (Fig. 7).

The main features presented for clusters with $2 N \leqslant 6$ were also observed for other clusters with larger number of particles. Different ground-state configurations appear when the number of particles increases, but the general behaviors of the systems shown before are the same, as can be seen in Fig. 8 for clusters with $2 N \leqslant 10$ [and $(d-2)=2$ ] as a function of $Z$. A larger number of regions were labeled, and the configurations associated with such new regions are presented in Fig. 9. The other regions-namely, (I),(II),...,(VIII)—are the same as the ones shown in Fig. 5.

\section{CONCLUSIONS}

We numerically studied the different structural configurations and the corresponding normal-mode spectrum of two vertically coupled 2D systems of interacting classical particles confined by a nonuniform distribution of charge. As an example the background charge was taken to be pointlike. These properties were analyzed as a function of the separation between the planes of charges $(d)$, as well as a function of the strength of the confinement potential $(Z)$. Our findings were illustrated for the $2 N \leqslant 6$ and $2 N \leqslant 10$ molecule systems. The $(d, Z)$ phase diagram exhibited a rich variety of first- and second-order structural phase transitions that delimit regions with symmetric and asymmetric configurations some of which are also associated with the unbinding of a particle.

In comparison to the previously studied parabolic vertically coupled dot systems by Partoens et al., ${ }^{16}$ novel asymmetrical ground-state configurations were found as well as evaporation of particles. The competition between the attrac- 
tive confinement potential and the repulsive interaction potential between the negative particles results in a rich and nontrivial set of equilibrium configurations.

The evaporation of one particle leads the system to an unbalanced distribution of charges over the planes and an asymmetrical ground-state configuration. Such a modification in the cluster is always characterized by a first-order structural transition.

The model system studied in the present paper also accounts for an overcharging which was found to be larger than the one found by Farias and Peeters ${ }^{8}$ in case of a single Coulomb-confined system. The screening of the interaction between charges in distinct layers is the cause of stability of the ionic state, defined here by an excess of the confined charges in the system.

The normal-mode spectrum is very sensitive to the symmetry of the ground-state configuration and to structural transitions observed in the system. In the former, a more degenerate spectrum is observed in contrast to the asymmetrical minimum-energy configurations. In the later, depending on the type of structural transition, a typical signature is observed in the normal-mode spectrum. For a firstorder structural transition there is a sudden jump in the normal-mode spectrum, while for a second-order structural transition there is a softening of one of the frequencies.

\section{ACKNOWLEDGMENTS}

F.F.M., W.P.F., and G.A.F. were supported by the Brazilian National Research Councils CNPq and CAPES. Part of this work was supported by the Flemish Science Foundation (FWO-Vl). *wandemberg@fisica.ufc.br

†rancois.peeters@ua.ac.be

${ }^{1}$ V. M. Bedanov and F. M. Peeters, Phys. Rev. B 49, 2667 (1994).

${ }^{2}$ V. A. Schweigert and F. M. Peeters, Phys. Rev. B 51, 7700 (1995).

${ }^{3}$ M. Saint Jean, C. Even, and C. Guthmann, Europhys. Lett. 55, 45 (2001).

${ }^{4}$ M. Kong, B. Partoens, and F. M. Peeters, Phys. Rev. E 65, 046602 (2002).

${ }^{5}$ W. P. Ferreira, A. Matulis, G. A. Farias, and F. M. Peeters, Phys. Rev. E 67, 046601 (2003).

${ }^{6}$ W. P. Ferreira, F. M. Peeters, and G. A. Farias, Phys. Rev. E 68, 066405 (2003).

${ }^{7}$ A. V. Filinov, M. Bonitz, and Yu. E. Lozovik, Phys. Rev. Lett. 86, 3851 (2001).

${ }^{8}$ G. A. Farias and F. M. Peeters, Solid State Commun. 100, 711 (1996).

${ }^{9}$ W. P. Ferreira, B. Partoens, F. M. Peeters, and G. A. Farias, Phys. Rev. E 71, 021501 (2005).

${ }^{10}$ H. Imamura, P. A. Maksym, and H. Aoki, Phys. Rev. B 59, 5817 (1999).

${ }^{11}$ S. Nagaraja, J.-P. Leburton, and R. M. Martin, Phys. Rev. B 60, 8759 (1999).

${ }^{12}$ P. A. Maksym, H. Imamura, G. P. Mallon, and H. Aoki, J. Phys.: Condens. Matter 12, R299 (2000).

${ }^{13}$ C. Yannouleas and U. Landman, Eur. Phys. J. D 16, 373 (2001).

${ }^{14}$ A. Harju, S. Siljamäki, and R. M. Nieminen, Phys. Rev. B 65,
075309 (2002)

${ }^{15}$ B. Szafran, F. M. Peeters, and S. Bednarek, Phys. Rev. B 70, 205318 (2004).

${ }^{16}$ B. Partoens, V. A. Schweigert, and F. M. Peeters, Phys. Rev. Lett. 79, 3990 (1997).

${ }^{17}$ M. Marlo, M. Alatalo, A. Harju, and R. M. Nieminen, Phys. Rev. B 66, 155322 (2002).

${ }^{18}$ R. Messina, C. Holm, and K. Kremer, Phys. Rev. E 64, 021405 (2001).

${ }^{19}$ F. M. Peeters, Phys. Rev. B 42, 1486 (1990).

${ }^{20}$ Z. L. Ye and E. Zaremba, Phys. Rev. B 50, 17217 (1994).

${ }^{21}$ B. P. van Zyl, E. Zaremba, and D. A. W. Hutchinson, Phys. Rev. B 61, 002107 (2000).

${ }^{22} \mathrm{H}$. Watanabe and T. Inoshita, Optoelectron., Devices Technol. 1, 33 (1986).

${ }^{23}$ T. Inoshita, S. Ohnishi, and A. Oshiyama, Phys. Rev. B 38, 3733 (1988); E. A. Andryushin and A. P. Silin, Fiz. Tverd. Tela (S.Peterburg) 33, 211 (1991) [Sov. Phys. Solid State 33, 123 (1991)].

${ }^{24}$ B. I. Shklovskii, Phys. Rev. Lett. 82, 3268 (1999).

${ }^{25}$ J. H. Chu and Lin I, Phys. Rev. Lett. 72, 4009 (1994); A. Melzer, Phys. Rev. E 67, 016411 (2003).

${ }^{26}$ B. A. Grzybowski, H. A. Stone, and G. M. Whitesides, Nature (London) 405, 1033 (2000); B. A. Grzybowski, X. Jiang, H. A. Stone, and G. M. Whitesides, Phys. Rev. E 64, 011603 (2001).

${ }^{27}$ R. Bubeck, C. Bechinger, S. Neser, and P. Leiderer, Phys. Rev. Lett. 82, 3364 (1999). 